\title{
Square root singularities of infinite systems of functional equations
}

\author{
Johannes F. Morgenbesser ${ }^{\dagger}$ \\ Institut für Diskrete Mathematik und Geometrie, Technische Universität Wien, Wiedner Hauptstraße 8-10, A-1040 \\ Wien, Austria
}

Infinite systems of equations appear naturally in combinatorial counting problems. Formally, we consider functional equations of the form $\mathbf{y}(x)=F(x, \mathbf{y}(x))$, where $F(x, \mathbf{y}): \mathbb{C} \times \ell^{p} \rightarrow \ell^{p}$ is a positive and nonlinear function, and analyze the behavior of the solution $\mathbf{y}(x)$ at the boundary of the domain of convergence. In contrast to the finite dimensional case different types of singularities are possible. We show that if the Jacobian operator of the function $F$ is compact, then the occurring singularities are of square root type, as it is in the finite dimensional setting. This leads to asymptotic expansions of the Taylor coefficients of $\mathbf{y}(x)$.

Keywords: functional equation, compact operator, Krein-Rutman theorem, infinite matrices, generating function

\section{Introduction}

Functional equations occur naturally in enumeration theory. For example, if $b(x)=\sum b_{n} x^{n}$ is the generating function of binary trees $\left(b_{n}\right.$ is the number of binary trees with $n$ internal nodes), then $b(x)$ satisfies $b(x)=1+x b(x)^{2}$. Indeed, if a combinatorial object has a recursive description, then the corresponding generating function $y(x)$ usually satisfies an equation of the form

$$
y(x)=F(x, y(x)) .
$$

Using this relation, it is often possible to extract the $n$-th coefficient (denoted by $\left[x^{n}\right] y(x)$ ) or the asymptotic behavior of $\left[x^{n}\right] y(x)$ (see for example [Drm09, FS09]). Under certain assumptions on $F$ (see Theorem 1 for a finite dimensional system), one can show that the solution of the functional equation has a so-called square root singularity on the boundary of the domain of convergence, i.e., $y(x)$ has a representation of the form

$$
y(x)=g(x)-h(x) \sqrt{1-\frac{x}{x_{0}}}
$$

\footnotetext{
${ }^{\dagger}$ The author is supported by the Austrian Science Foundation FWF, grant S9604, that is part of the National Research Network "Analytic Combinatorics and Probabilistic Number Theory".
}

1365-8050 (C) 2010 Discrete Mathematics and Theoretical Computer Science (DMTCS), Nancy, France 
locally around some point $x_{0}$, where $g(x)$ and $h(x)$ are analytic functions. If $y(x)$ has an analytic continuation to a $\Delta$-domain $\Delta\left(x_{0}, \eta, \delta\right)=\left\{x:|x|<x_{0}+\eta,\left|\arg \left(x / x_{0}-1\right)\right|>\delta\right\}$, then the Transfer Lemma of Flajolet and Odlyzko (see [FO90]) implies that

$$
\left[x^{n}\right] y(x) \sim \frac{c}{x_{0}^{n} n^{3 / 2}},
$$

where $c$ is a positive constant. Drmota, Lalley and Woods (see [Drm97, La193, La101, Woo97]) generalized this result, based on the idea of using systems of functional equations. For example, Drmota proved the following theorem ( $c f$. [BBY09, Drm09]):

Theorem 1 Let $F(x, \mathbf{y})=\left(F_{1}(x, \mathbf{y}), \ldots, F_{N}(x, \mathbf{y})\right)$ be a nonlinear system of functions analytic around $x=0$ and $\mathbf{y}=\left(y_{1}, \ldots, y_{N}\right)=\mathbf{0}$, whose Taylor coefficients are all nonnegative, such that $F(0, \mathbf{y})=$ $0, F(x, \mathbf{0}) \neq 0$ and $F_{x}(x, \mathbf{y}) \neq 0$. Furthermore assume that the dependency graph ${ }^{(\mathrm{i})}$ of $F$ is strongly connected and that the region of convergence of $F$ is large enough such that the system

$$
\mathbf{y}=F(x, \mathbf{y}), \quad r\left(\frac{\partial F}{\partial \mathbf{y}}(x, \mathbf{y})\right)=1,
$$

has a real and positive solution $\left(x_{0}, \mathbf{y}_{0}\right)$ in its interior, where $r(\partial F / \partial \mathbf{y}(x, \mathbf{y}))$ denotes the spectral radius of the Jacobian matrix. Let $\mathbf{y}(x)$ denote the solution of the system $\mathbf{y}=F(x, \mathbf{y})$ with $\mathbf{y}(0)=\mathbf{0}$. Then there exists $\varepsilon>0$ such that the functions $y_{j}(x)$ admit a representation of the form

$$
y_{j}(x)=g_{j}(x)-h_{j}(x) \sqrt{1-\frac{x}{x_{0}}}
$$

for $\left|x-x_{0}\right|<\varepsilon$ and $\arg \left(x-x_{0}\right) \neq 0$, where $g_{j}$ and $h_{j}$ are analytic functions.

Here, it is important that the system of functional equations is nonlinear. Indeed, in the linear case, one easily obtains that the singularity can only be a simple pole. Thus, for finite dimensional and strongly connected systems there can only occur square root singularities or poles.

The main goal of this work is to consider infinite systems of functional equations. Actually, the study of singularities in the infinite dimensional setting is much more involved. Even in the case of linear infinite systems, there can arise different types of singularities, as the following examples show (see [Pro04] for details): Let $F: \mathbb{C} \times \ell^{\infty} \rightarrow \ell^{\infty}$ be defined by

$$
\begin{aligned}
& F_{1}(x, \mathbf{y})=1+x y_{2}, \\
& F_{i}(x, \mathbf{y})=x y_{i-1}+x y_{i+1}, \quad(i \geqslant 2),
\end{aligned}
$$

where $\mathbf{y}=\left(y_{1}, y_{2}, \ldots\right)$. This function can also be written in the form $F(x, \mathbf{y})=A(x) \mathbf{y}+\mathbf{b}$, where $A(x)$ is the linear operator

$$
A(x)=\left(\begin{array}{ccccccc}
0 & x & 0 & 0 & \ldots & & \\
x & 0 & x & 0 & \ldots & & \\
0 & x & 0 & x & 0 & \ldots & \\
0 & 0 & x & 0 & x & 0 & \ldots \\
\vdots & \vdots & \ddots & \ddots & \ddots & \ddots & \ddots
\end{array}\right)
$$

\footnotetext{
(i) The dependency graph $G=(V, E)$ is defined in the following way: The vertices $V=\left(y_{1}, \ldots, y_{N}\right)$ are the unknown functions and an ordered pair $\left(y_{i}, y_{j}\right)$ is contained in the edge set $E$ if and only if $\partial F_{i} / \partial y_{j} \neq 0$ (i.e., $F_{i}$ really depends on $y_{j}$ ).
} 
and $\mathbf{b}=(1,0,0, \ldots)$. The function $\mathbf{y}(x)=\left(y_{1}(x), y_{2}(x), \ldots\right)$ defined by

$$
y_{i}(x)=\frac{1}{x}\left(\frac{1-\sqrt{1-4 x^{2}}}{2 x}\right)^{i}
$$

is the unique and analytic solution of the equation $\mathbf{y}(x)=F(x, \mathbf{y}(x))$ with $\mathbf{y}(0)=\mathbf{b}$. The functions $y_{j}(x)$ have a square root singularity at $x=1 / 2$ since $y_{i}(x)=g_{i}(x)-h_{i}(x) \sqrt{1-2 x}$, where $g_{i}(x)$ and $h_{i}(x)$ are given by

$$
g_{i}(x)=\frac{1}{2^{i} x^{i+1}} \sum_{k=0}^{\left\lfloor\frac{i}{2}\right\rfloor}\left(\begin{array}{c}
i \\
2 k
\end{array}\right)\left(1-4 x^{2}\right)^{k} \quad \text { and } \quad h_{i}(x)=\frac{\sqrt{1+2 x}}{2^{i} x^{i+1}} \sum_{k=0}^{\left\lfloor\frac{i-1}{2}\right\rfloor}\left(\begin{array}{c}
i \\
2 k+1
\end{array}\right)\left(1-4 x^{2}\right)^{k} .
$$

This example is related to nonnegative lattice paths and appeared as an exercise in Knuth's book [Knu75]. As a second example we consider a linear system that is related to so-called Knödel walks. Let $F$ : $\mathbb{C} \times \ell^{\infty} \rightarrow \ell^{\infty}$ be defined by

$$
\begin{aligned}
& F_{1}(x, \mathbf{y})=x y_{2}, \\
& F_{2}(x, \mathbf{y})=1+x y_{1}+x y_{3} \\
& F_{3}(x, \mathbf{y})=x y_{1}+x y_{2}+x y_{4} \\
& F_{i}(x, \mathbf{y})=x y_{i-1}+x y_{i+1}, \quad(i \geqslant 4) .
\end{aligned}
$$

Again, there exists a solution $\mathbf{y}=\mathbf{y}(x)$ of the functional equation $\mathbf{y}(x)=F(x, \mathbf{y}(x))$ (with $\mathbf{y}(0)=$ $(0,1,0,0, \ldots))$. However, this time there is a singularity of the form $(1-2 x)^{-1 / 2}$ at $x=1 / 2$.

Also in the case of nonlinear systems different types of singularities are possible. The next example is related to the vertical profile of trees ( $c f$. Bousquet-Mélou [BM06] and Bouttier et al. [BDFG03], see also [Drm09, Section 5.1]). Let $F: \mathbb{C} \times \ell^{\infty} \rightarrow \ell^{\infty}$ be defined by

$$
\begin{aligned}
& F_{1}(x, \mathbf{y})=0 \\
& F_{i}(x, \mathbf{y})=1+x y_{i}\left(y_{i-1}+y_{i}+y_{i+1}\right), \quad(i \geqslant 2) .
\end{aligned}
$$

This system leads to a solution $\mathbf{y}(x)$ that has a dominant singularity of the form $(1-12 x)^{3 / 2}$. Another nonlinear system was studied by Lalley. In his work on random walks on infinite free products of groups [Lal02] he considered the operator (acting on $\ell^{1}$ ) that is related to the recursive system

$$
F_{i ; x}(z)=z\left\{p_{i} q_{x}+p_{\emptyset} F_{i ; x}(z)+\sum_{y \in \Gamma_{i} \backslash\{x\}} p_{i} q_{y} F_{i ; y^{-1} x}(z)+\sum_{j \neq i} \sum_{y \in \Gamma_{i}} p_{j} q_{y} F_{j ; y^{-1}}(z) F_{i ; x}(z)\right\},
$$

where $\Gamma_{i}, i \geqslant 1$ are finite groups and $p_{i}$ and $q_{x}$ are certain probabilities. It turns out that in this case there is a square root singularity. Note, that the Jacobian operator of the corresponding function $F$ is the sum of a compact operator and a scalar multiple of the identity (see [Lal02, Lemma 4.1]). 


\section{General Setting and Main Theorem}

We have seen in the preceding section that infinite dimensional systems do not behave in general like finite dimensional ones. In this work we want to show that under the additional assumption of compactness of the Jacobian operator of $F$ we indeed obtain quite the same behavior as for finite systems of functional equations.

Before we state the main result, we recall some definitions from the field of functional analysis in order to be able to specify the basic setting. Let $B$ be a Banach space and $U$ the open unit ball in $B$. An operator $T: B \rightarrow B$ is compact, if the closure of $T(U)$ is compact in $B$ (or, equivalently, if every bounded sequence $\left(x_{n}\right)_{n \geqslant 0}$ in $B$ contains a subsequence $\left(x_{n_{i}}\right)_{i \geqslant 0}$ such that $\left(T x_{n_{i}}\right)_{i \geqslant 0}$ converges in $\left.B\right)$. A function $F: B \rightarrow B$ is called Fréchet differentiable at $x_{0}$ if there exists a bounded linear operator $(\partial F / \partial x)\left(x_{0}\right)$ such that

$$
F\left(x_{0}+h\right)=F\left(x_{0}\right)+\frac{\partial F}{\partial x}\left(x_{0}\right) h+\omega\left(x_{0}, h\right) \quad \text { and } \quad \omega\left(x_{0}, h\right)=o(\|h\|), \quad(h \rightarrow 0) .
$$

The operator $\partial F / \partial x$ is called the Fréchet derivative of $F$. If $B$ is a complex vector space and (1) holds for all $h$, then $F$ is said to be analytic in $x_{0} . F$ is analytic in $\Omega \subseteq B$, if it is analytic for all $x_{0} \in \Omega$. Analyticity is equivalent to the fact that for all $x_{0} \in \Omega$ there exist an $r>0$ and continuous symmetric $n$-linear forms $A_{n}$ such that $\sum_{n \geqslant 1}\left\|A_{n}\right\| r^{n}<\infty$ and

$$
F\left(x_{0}+h\right)=F\left(x_{0}\right)+\sum_{n \geqslant 1} \frac{A_{n}}{n !}\left(h^{n}\right)
$$

in a neighborhood of $x_{0}$ (see [Dei85, Section 7.7 and 15.1]). (The "coefficients" $A_{n}$ are equal to the (iteratively defined) $n$-th Fréchet derivatives of $F$ ).

In what follows, we mainly deal with the Banach space $\ell^{p}=\ell^{p}(\mathbb{N})(1 \leqslant p<\infty)$ of all complex valued sequences $\left(t_{n}\right)_{n \in \mathbb{N}}$ satisfying $\left\|\left(t_{n}\right)\right\|_{p}^{p}:=\sum_{n=1}^{\infty}\left|t_{n}\right|^{p}<\infty$. (The space $\ell^{\infty}=\ell^{\infty}(\mathbb{N})$ is the space of all bounded complex sequences $\left(z_{n}\right)$ with norm $\left\|\left(z_{n}\right)\right\|_{\infty}=\sup _{n \geqslant 1}\left|z_{n}\right|<\infty$.) In this case, the Fréchet derivative is also called Jacobian operator (in analogy to the finite dimensional case). We call a function $F: \mathbb{C} \times \ell^{p} \rightarrow \ell^{p}$ positive (in $U \times V$ ), if there exist nonnegative real numbers $a_{i \mathbf{j}, k}$ such that for all $k \geqslant 1$ and for all $(x, \mathbf{y}) \in U \times V$,

$$
F_{k}(x, \mathbf{y})=\sum_{i, \mathbf{j}} a_{i \mathbf{j}, k} x^{i} y^{\mathbf{j}},
$$

where $\mathbf{j} \in \mathbb{N}^{\mathbb{N}}$, only finitely many components are nonzero, and $y^{\mathbf{j}}=y_{1}^{j_{1}} y_{2}^{j_{2}} y_{3}^{j_{3}} \cdots$.

As already indicated above, the compactness of the Jacobian operator $\partial F / \partial \mathbf{y}$ will play a crucial role in our considerations. Moreover, we have to assume that $\partial F / \partial \mathbf{y}$ is irreducible. In order to be able to define this property, we recall some basic notion from functional analysis on $\ell^{p}$ spaces. Any bounded linear operator on an $\ell^{p}$ space $(1 \leqslant p<\infty)$ is uniquely determined by an infinite dimensional matrix $\left(a_{i j}\right)_{1 \leqslant i, j<\infty}$ via the equation

$$
(A x)_{i}=\sum_{k=1}^{\infty} a_{i k} x_{k},
$$

where $\left(x_{k}\right)_{1 \leqslant k<\infty}$ is written with respect to the canonical standard bases in $\ell^{p}$. We call the matrix $\left(a_{i j}\right)_{1 \leqslant i, j<\infty}$ the matrix representation of $A$ (and write $A=\left(a_{i j}\right)_{1 \leqslant i, j<\infty}$ or just $A=\left(a_{i j}\right)$ ). An 
operator $A$ is called positive, if all entries of the matrix representation of $A$ are nonnegative. A positive operator $A=\left(a_{i j}\right)$ is said to be irreducible, if for every pair $(i, j)$ there exists an integer $n=n(i, j)>0$, such that $a_{i j}^{(n)}>0$, where

$$
A^{n}=\left(a_{i j}^{(n)}\right)_{1 \leqslant i, j<\infty} .
$$

Finally, if $A$ is a bounded operator on a Banach space, then $r(A)$ denotes the spectral radius of $A$ (i.e., $r(A)=\sup _{\lambda \in \sigma(A)}|\lambda|$, and $\sigma(A)$ is the spectrum of $A$ ).

Theorem 2 Let $1 \leqslant p<\infty$ and $F: \mathbb{C} \times \ell^{p} \rightarrow \ell^{p},(x, \mathbf{y}) \mapsto F(x, \mathbf{y})$ be an analytic and positive function defined in an open neighborhood $U \times V$ of $(0,0)$ satisfying:

(i) $F(0, \mathbf{y})=\mathbf{0}$ for all $\mathbf{y} \in V$,

(ii) $F(x, \mathbf{0}) \not \equiv \mathbf{0}$ in $U$,

(iii) $\frac{\partial F}{\partial \mathbf{y}}(x, \mathbf{y})$ is a compact operator on $\ell^{p}$ for all $(x, \mathbf{y}) \in U \times V$ and irreducible for strictly positive $(x, \mathbf{y}) \in U \times V$.

Furthermore, assume that the system

$$
\begin{aligned}
& \mathbf{y}=F(x, \mathbf{y}), \\
& r\left(\frac{\partial F}{\partial \mathbf{y}}(x, \mathbf{y})\right)=1,
\end{aligned}
$$

has a positive solution $\left(x_{0}, \mathbf{y}_{0}\right) \in U \times V$. Then there exists an analytic solution of

$$
\mathbf{y}=F(x, \mathbf{y})
$$

with $\mathbf{y}(0)=\mathbf{0}$ such that the following holds: There exists $\varepsilon>0$ such that $\mathbf{y}(x)$ admits a representation of the form

$$
\mathbf{y}(x)=\mathbf{g}(x)-\mathbf{h}(x) \sqrt{1-\frac{x}{x_{0}}}
$$

for $\left|x-x_{0}\right|<\varepsilon$ and $\arg \left(x-x_{0}\right) \neq 0$, where $\mathbf{g}(x)$ and $\mathbf{h}(x)$ are analytic functions.

Moreover, if there exist two integers $n_{1}$ and $n_{2}$ that are relatively prime such that $\left[x^{n_{1}}\right] y_{1}(x)>0$ and $\left[x^{n_{2}}\right] y_{1}(x)>0$, then $x_{0}$ is the only singularity of $\mathbf{y}(x)$ on the circle $|x|=x_{0}$ and we obtain for every $j \geqslant 1$ an asymptotic expansion for $\left[x^{n}\right] y_{j}(x)$ of the form

$$
\left[x^{n}\right] y_{j}(x) \sim \frac{c_{j}}{x_{0}^{n} n^{3 / 2}}
$$

where $c_{j}$ is a positive constant.

Remark 1 Lalley [Lal02] uses a different method in his work on random walks in order to study the dominating singularity of the system stated in Section 1. In the proof of Theorem 2 given below (see Section 5), we use a reduction of the infinite system of functional equations which allows us to take advantage of the positivity assumptions (see Section 4). This makes the Theorem more generally applicable, since we directly obtain asymptotic expansions of the Taylor coefficients. 
Remark 2 Let $F$ be a linear function (or more precisely an affine map), i.e. $F(x, \mathbf{y})=A(x) \mathbf{y}+\mathbf{b}(x)$, where $A(x)$ is a linear operator and $\mathbf{b}(x)$ a vector in $\ell^{p}$. Then it follows (under the assumption that $A(x)$ is positive and compact, $F(0, \mathbf{y})=\mathbf{0}$ and $F(x, \mathbf{0}) \not \equiv \mathbf{0})$ that

$$
\mathbf{y}(x)=(I-A(x))^{-1} \mathbf{b}(x)
$$

is a solution of the functional equation $\mathbf{y}=F(x, \mathbf{y})$ as long as the spectral radius of $A(x)$ is smaller than 1. Furthermore, the resolvent operator $(I-A(x))^{-1}$ has a pole if $r(A(x))=1$. Hence, in the case of compact Jacobian operators we obtain the same types of singularities as in the finite dimensional case. (The linear operators in Section 1 are clearly not compact). Note, that the assumptions on $F$ and the existence of the solution $\left(x_{0}, \mathbf{y}_{0}\right)$ satisfying (2) imply that we are actually dealing with nonlinear functions in Theorem 2.

Remark 3 The examples in Section 1 show that the assumption of compactness of the Jacobian operator cannot be canceled without stating any other restrictions on $F$. In order to prove Theorem 2, we use excessively the special structure of the spectrum and the resolvent of compact operators. It seems possible to extend the result to operators which have similar spectral properties as compact operators (for example Riesz operators). In the example of Lalley, the spectrum of the Jacobian operator is a shifted spectrum of a compact operator. Even though Theorem 2 does not include such cases, a similar argumentation should imply the desired result. The Jacobian operator in the example of Bousquet-Mélou and Bouttier et al. is "far away" from being compact. And indeed, they even get a dominant singularity different from the one stated in our theorem.

Remark 4 In order to use this theorem for showing central limit results, one has to include an additional continuous parameter similar to the parameter $\mathbf{u}$ in [Drm09, Theorem 2.33] (i.e., $F$ is of the form $F$ : $\mathbb{C} \times \ell^{p} \times \ell^{p} \rightarrow \ell^{p},(x, \mathbf{y}, \mathbf{u}) \mapsto F(x, \mathbf{y}, \mathbf{u})$ and the functional equation has solutions $\left.\mathbf{y}=\mathbf{y}(x, \mathbf{u})\right)$.

\section{Tree counting problems with degree restrictions}

In this section we apply Theorem 2 to a counting problem. Our combinatorial objects are rooted labeled trees. More precisely, if $T$ is a tree of size $n$ (i.e., a tree with $n$ nodes), then all nodes are labeled by $1,2, \ldots, n$ and there exists a specific node that is marked (the root).

Let $\mathcal{T}_{n}$ be the set of all rooted labeled trees of size $n$ with the property that each node with out-degree $k$ has successors with out-degree $k-1$ or $k+1$ (see Figure 1). The first few members of the sequence $\left(\# \mathcal{T}_{n}\right)_{n \geqslant 1}$ are given by $(1,2,0,0,60,360,0, \ldots)$.

In what follows, we show that there exist positive constants $c$ and $x_{0}$ with $1 / e \leqslant x_{0} \leqslant 1$ such that ${ }^{\text {(ii) }}$

$$
\# \mathcal{T}_{n} \sim \frac{c}{x_{0}^{n} n^{3 / 2}} n ! .
$$

Note, that such a result is quite natural in this context (cf. [BBY06], where it is shown that a law of the form $c x_{0}^{-n} n^{-3 / 2}$ occurs frequently when counting rooted trees.) Let $y_{k}(x)$ be the exponential generating function

$$
y_{k}(x)=\sum_{n=0}^{\infty} \frac{b_{k n}}{n !} x^{n},
$$

\footnotetext{
(ii) This example can be easily generalized to families of trees with other degree restrictions.
} 

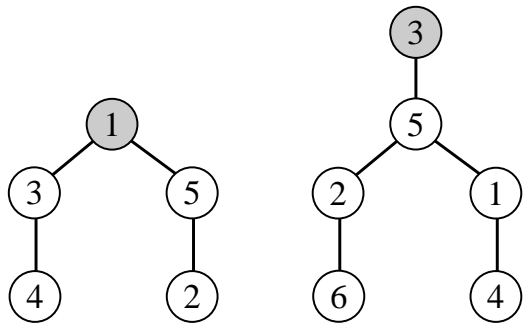

Fig. 1: Two considered trees of size 5 and 6 (the roots are marked gray)

where $b_{k n}$ counts the number of trees $T$ in $\mathcal{T}_{n}$, such that the root has degree $k$. Note, that we have $y_{0}(x)=x$. The recursive structure of $\mathcal{T}_{n}$ easily implies that the functions $y_{k}(x)$ satisfy

$$
y_{k}(x)=x \frac{\left(y_{k-1}(x)+y_{k+1}(x)\right)^{k}}{k !}
$$

for all $k \geqslant 1$. Hence, $\mathbf{y}(x)=\left(y_{1}(x), y_{2}(x), \ldots\right)$ satisfies the functional equation $\mathbf{y}(x)=F(x, \mathbf{y}(x))$, where $F: \mathbb{C} \times \ell^{1} \rightarrow \ell^{1}$ is defined by

$$
F_{1}(x, \mathbf{y})=x\left(y_{2}+x\right) \quad \text { and } \quad \mathrm{F}_{k}(x, \mathbf{y})=x \frac{\left(y_{k-1}+y_{k+1}\right)^{k}}{k !}, \quad(k \geqslant 2) .
$$

First, we have to show that $F$ is indeed a function from $\mathbb{C} \times \ell_{1}$ to $\ell_{1}$. Let $\mathbf{y}=\left(y_{1}, y_{2}, \ldots\right) \in \ell^{1}$. Then we have

$$
\begin{aligned}
\sum_{k=1}^{\infty}\left|F_{k}(x, \mathbf{y})\right| & =|x|\left|y_{2}+x\right|+|x| \sum_{k=2}^{\infty} \frac{\left|y_{k-1}+y_{k+1}\right|^{k}}{k !} \\
& \leqslant|x|\left|y_{2}\right|+|x|^{2}+|x| \sum_{k=2}^{\infty} \frac{2^{k}\left(\left|y_{k-1}\right|^{k}+\left|y_{k+1}\right|^{k}\right)}{k !} \\
& \leqslant|x|\|\mathbf{y}\|_{\infty}+|x|^{2}+2|x| \sum_{k=2}^{\infty} \frac{\left(2\|\mathbf{y}\|_{\infty}\right)^{k}}{k !} \\
& \leqslant|x|\left(\|\mathbf{y}\|_{\infty}+|x|+2 e^{2\|\mathbf{y}\|_{\infty}}\right)<\infty
\end{aligned}
$$

Actually, we have shown that $F$ maps $\mathbb{C} \times \ell_{\infty}$ to $\ell_{1}$. Clearly, $F$ is analytic and we have $F(0, \mathbf{y})=0$ and $F(x, 0) \not \equiv 0$. A similar calculation as in (6) shows that $\partial F / \partial \mathbf{y}$ is an operator from $\ell^{1}$ to $\ell^{1}$. Furthermore, $\partial F / \partial \mathbf{y}$ is irreducible. Since this matrix can be approximated with respect to the $\ell_{1}$-operator norm by finite rank operators (take the first $n$ columns of $\partial F / \partial \mathbf{y}$ ), we obtain that $\partial F / \partial \mathbf{y}$ is compact.

In order to apply Theorem 2 , we have to show that there exists $\left(x_{0}, \mathbf{y}_{0}\right)$ satisfying $\mathbf{y}_{0}=F\left(x_{0}, \mathbf{y}_{0}\right)$ and $r\left(\partial F / \partial \mathbf{y}\left(x_{0}, \mathbf{y}_{0}\right)\right)=1$. We know from the Implicit Function Theorem that there exists a positive solution $\mathbf{y}(x)$ of the functional equation around $x=0$ (compare with the proof of Theorem 2). Let $x_{0}$ be the smallest positive real number such that $\mathbf{y}(x)$ cannot be analytically continued in $x_{0}$ (combinatorial considerations show that $\left.1 / e \leqslant x_{0} \leqslant 1\right)$. Consider the function $\mathbf{y}(x)$ for $x \rightarrow x_{0}$. The spectral radius 
of the Jacobian operator is 0 for $x=0$ and it is continuous (see Lemma 2) and monotone increasing if $x$ increases (see Proposition 2 and note that $\mathbf{y}(x)$ is positive). Moreover, we have $r(\partial F / \partial \mathbf{y}(x, \mathbf{y}(x)))<1$ for $x<x_{0}$. Indeed, if $r(\partial F / \partial \mathbf{y}(x, \mathbf{y}(x)))=1$, then $\mathbf{y}(x)$ cannot be analytic in an neighborhood of $x$, which is in contrast to the fact that $x<x_{0}$ (implicit differentiation yields a contradiction). This implies that $\mathbf{y}(x)$ cannot have a pole (in any of its components) at $x_{0}$ and $\mathbf{y}(x)$ converges to some positive vector $\mathbf{y}_{0}$ for $x \rightarrow x_{0}$. A priori, $\mathbf{y}_{0}$ is in $\ell^{\infty}$. But since $\mathbf{y}_{0}$ also satisfies the functional equation, it is also in $\ell_{1}$ (see (6)). Furthermore, we have $r\left(\partial F / \partial \mathbf{y}\left(x_{0}, \mathbf{y}_{0}\right)\right)=1$ since we could continue $\mathbf{y}(x)$ otherwise in an neighborhood of $x_{0}$.

Thus, all conditions of Theorem 2 are satisfied and we obtain that $\mathbf{y}(x)$ has a square root singularity. Since $\mathbf{y}(x) \in \ell^{1}$, we have that $t(x)=\sum_{k=0}^{\infty} y_{k}(x)$ has a square root singularity, too. Since $t(x)$ can also be written in the form $t(x)=\sum_{n=0}^{\infty} \frac{\# \mathcal{T}_{n}}{n !} x^{n}$, this finally implies (5).

\section{Monotonicity of spectral radii}

In this section we will show some spectral properties of compact and positive operators on $\ell^{p}$ spaces. Recall, that any bounded linear operator $A$ on $\ell^{p}(1 \leqslant p<\infty)$ is uniquely determined by an infinite dimensional matrix $\left(a_{i j}\right)_{1 \leqslant i, j<\infty}$.

The study of operators (or matrices) in $\ell^{\infty}$ is different. First note, that there is no one-to-one correspondence between operators and matrices. (Actually, there exist nontrivial compact operators, such that the corresponding "matrix representation" is the zero matrix). Nevertheless, if we have a matrix $\left(a_{i j}\right)_{1 \leqslant i, j<\infty}$, we define an operator $A$ on $\ell^{\infty}$ via

$$
(A x)_{i}=\sum_{k=1}^{\infty} a_{i k} x_{k},
$$

if the summation is well-defined for all $i \geqslant 1$ and for all $x \in \ell^{\infty}$. Recall that an operator $A$ is called positive if all entries of the matrix representation of $A$ are nonnegative. If $u$ and $v$ are real vectors or matrices, $u \geqslant v$ means that all entries of $u$ are greater than or equal to the corresponding entries of $v$. Thus, an operator $A$ is positive if $\left(a_{i j}\right) \geqslant 0$. Similarly, a vector $x$ is called positive (or also nonnegative) if $x \geqslant 0$. We call $x$ strictly positive, if all entries $x_{i}$ of $x$ satisfy $x_{i}>0$. Moreover, if $u$ is a vector with entries $u_{i}$, then $|u|$ denotes the vector with entries $\left|u_{i}\right|$ (a similar definition is used for matrices).

The adjoint operator of an operator $A$ (denoted by $A^{*}$ ) is acting on $\ell^{p^{\prime}} \cong \ell^{q}$, where $1 / p+1 / q=1$ (for $1 \leqslant p<\infty)$. The operator $A^{*}$ can be associated with the matrix $\left(a_{j i}\right)_{1 \leqslant i, j<\infty}$ acting on $\ell^{q}$ (which we do in the sequel without explicitly saying so).

The following result goes back to Kreı̆n and Rutman [KR50] and is of particular importance for our work (see [Zei86, Proposition 7.26]):

Lemma 1 Let $T=\left(t_{i j}\right)_{1 \leqslant i, j<\infty}$ be a compact positive operator on $\ell^{p}$ (where $1 \leqslant p<\infty$ ) and assume that $r(T)>0$. Then $r(T)$ is an eigenvalue of $T$ with nonnegative eigenvector $\beta \in \ell^{p}$. Moreover, $r(T)=r\left(T^{*}\right)$ is an eigenvector of $T^{*}$ with nonnegative eigenvector $\alpha \in \ell^{q}$.

Proposition 1 Let $A=\left(a_{i j}\right)_{1 \leqslant i, j<\infty}$ be a positive, irreducible and compact operator on $\ell^{p}, 1 \leqslant p<\infty$. Let $B=\left(b_{i j}\right)_{1 \leqslant i, j<\infty}$ be defined by $b_{i j}=a_{i+1} j+1$. Then we have

$$
r(B)<r(A) \text {. }
$$


Remark 5 The matrix $B$ is obtained through eliminating the first row and first column of $A$. The same result holds, if one removes a finite number of rows and (the corresponding) columns of $A$.

Before we prove this proposition, we recall some notation concerning infinite matrices (see [VJ67]). A nonnegative vector $x \neq 0$ (lying in the sequence space of real numbers) is called left (or right) $r$ subinvariant vector of $\left(a_{i j}\right)$, if for all $j \geqslant 1$

$$
r \sum_{k=1}^{\infty} a_{k j} x_{k} \leqslant x_{j} \quad \text { or } \quad r \sum_{k=1}^{\infty} a_{j k} x_{k} \leqslant x_{j},
$$

respectively. On the other hand, $x \neq 0$ is called left (or right) $r$-superinvariant vector of $\left(a_{i j}\right)$, if for all $j \geqslant 1$

$$
r \sum_{k=1}^{\infty} a_{k j} x_{k} \geqslant x_{j} \quad \text { or } \quad r \sum_{k=1}^{\infty} a_{j k} x_{k} \geqslant x_{j},
$$

respectively. The vector $x$ is called (left or right) $r$-invariant, if it is (left or right) $r$-sub- and superinvariant.

Proof of Proposition 1: It easily follows from the irreducibility condition that $r(A)>0$. Let $m$ be an integer such that $d=a_{11}^{(m)}>0$. Then we have $\left\|A^{m n}\right\| \geqslant d^{n}$ for all $n \geqslant 1$, where $\|\cdot\|$ denotes the operator norm that is induced by the $p$-norm on $\ell^{p}$ (consider $A^{m} e_{1}$, where $e_{1}=(1,0,0, \ldots)$ ). Gelfand's formula implies $r(A)=\lim _{n \rightarrow \infty}\left\|A^{n}\right\|^{1 / n} \geqslant d^{1 / m}$. If $r(B)=0$ (note that $B$ need not to be irreducible), the statement of the result follows from the fact that $r(A)>0$. Suppose now that $r(B)>0$.

Let $S_{\ell}$ be the left shift operator and $S_{r}$ the right shift operator acting on $\ell^{p}$ (i.e., $S_{\ell}\left(x_{1}, x_{2}, x_{3}, \ldots\right)=$ $\left(x_{2}, x_{3}, \ldots\right)$ and $\left.S_{r}\left(x_{1}, x_{2}, \ldots\right)=\left(0, x_{1}, x_{2}, \ldots\right)\right)$. Then $B=S_{\ell} A S_{r}$. Since the shift operators are bounded and $A$ is compact, it follows that $B$ is also compact. Moreover, $B$ is clearly a positive operator. Hence, the assumptions of Lemma 1 are satisfied and there exists a nonnegative eigenvector $y$ of $B$ to the eigenvalue $r(B)$. Set $x:=S_{r} y$. Then we have

$$
A x=(\star, 0, \ldots)+r(B) x \geqslant r(B) x,
$$

where $\star$ is some positive number. Thus, $x$ is a right $1 / r(B)$-superinvariant vector of $\left(a_{i j}\right)$. If we assume that $r(B) \geqslant r(A)$, then $x$ is also a right $1 / r(A)$-superinvariant vector. According to Lemma $1, A$ has a nonnegative left eigenvector $\alpha \in \ell^{q}$ to the eigenvalue $r(A)$. This vector is clearly a left $1 / r(A)$ subinvariant vector (in particular, it is also strictly positive, see [VJ67, Lemma 4.1]). Hölders inequality and the fact that $x \in \ell^{p}$ imply

$$
\sum_{n=1}^{\infty} x_{n} \alpha_{n}<\infty
$$

It follows from [VJ67, Lemma 5.2] that this is only possible, if $x=0$ or $x$ is strictly positive and $1 / r(A)$ invariant. But since $x_{1}=0$ and $x \neq 0$, the assumption $r(B) \geqslant r(A)$ cannot hold true and Proposition 1 is shown.

Proposition 2 Let $1 \leqslant p<\infty$ and $A=\left(a_{i j}\right)_{1 \leqslant i, j<\infty}$ and $C=\left(c_{i j}\right)_{1 \leqslant i, j<\infty}$ be compact operators on $\ell^{p}$. Furthermore, let $A$ be positive and irreducible such that $|C| \leqslant A$ but $|C| \neq A$. Then we have

$$
r(C)<r(A)
$$


Proof: The proof is similar to the proof of Proposition 1. First we have that $r(A)>0$. If $r(C)=0$, we are done. Assume now that $r(C)>0$. Since $C$ is compact, there exists an eigenvector $y \in \ell^{p}$ to some eigenvalue $s$ with $|s|=r(C)$. We get

$$
|s y|=r(C)|y| \leqslant|C||y| \leqslant A|y| .
$$

If we assume that $r(C) \geqslant r(A)$, then $|y|$ is a right $1 / r(A)$-superinvariant vector. The same reasoning as above shows that this is only possible, if $|y|=0$ (which is clearly not true) or if $|y|$ is strictly positive and $1 / r(A)$-invariant. In the case of $1 / r(A)$-invariance, we obtain

$$
A|y|=r(A)|y| \leqslant r(C)|y| \leqslant|C||y| \leqslant A|y| .
$$

Thus, we have $(A-|C|)|y|=0$. But since $|y|$ is strictly positive and $A \neq|C|$, this is impossible.

\section{Proof of Theorem 2}

Before we prove our main theorem, we show that the spectral radius of the Jacobian operator of $F$ is continuous.

Lemma 2 Let the function $F$ satisfy the assumptions of Theorem 2. Then we have that the map

$$
(x, \mathbf{y}) \mapsto r\left(\frac{\partial F}{\partial \mathbf{y}}(x, \mathbf{y})\right)
$$

is continuous for all $(x, \mathbf{y}) \in U \times V$.

Proof: First note, that $(x, \mathbf{y}) \mapsto \frac{\partial F}{\partial \mathbf{y}}(x, \mathbf{y})$ is continuous. Let $(x, \mathbf{y}) \in U \times V$ such that $r\left(\frac{\partial F}{\partial \mathbf{y}}(x, \mathbf{y})\right)>0$. Since isolated eigenvalues with finite multiplicity must vary continuously (see [Kat66, Chapter IV.3.5]) and since we are dealing with compact operators, we obtain the desired result. If $r\left(\frac{\partial F}{\partial \mathbf{y}}(x, \mathbf{y})\right)=0$ (as it is for example in $(0, \mathbf{0}))$, then the continuity follows from the upper semicontinuity of the spectrum of closed operators (see [Kat66, Chapter IV.3.1]).

Proof of Theorem 2: The Implicit Function Theorem (see [Dei85, Theorem 15.3]) implies that there exists a unique analytic solution $\mathbf{y}=\mathbf{y}(x)$ of the functional equation (3) in a neighborhood of $(0, \mathbf{0})$. It also follows from the Banach Fixed-Point Theorem that the sequence $y_{0} \equiv 0$ and

$$
y_{n+1}(x)=F\left(x, y_{n}(x)\right), \quad n \geqslant 1,
$$

converges uniformly to the unique solution $\mathbf{y}(x)$ of (3). Thus $\mathbf{y}(x)$ is positive. Since the Jacobian operator is continuous and $F$ is positive, we have that the spectral radius of the Jacobian operator is less than 1 for all $(x, \mathbf{y}) \in U \times V$ with $|x|<x_{0}$ and $|y|<\mathbf{y}_{0}$ (cf. Proposition 2). The Implicit Function Theorem implies that the positive function $\mathbf{y}(x)$ is actually defined for all $|x|<x_{0}$. Next, we divide equation (2) up into two equations

$$
\begin{array}{r}
y_{1}=F_{1}\left(x, y_{1}, \overline{\mathbf{y}}\right), \\
\overline{\mathbf{y}}=\bar{F}\left(x, y_{1}, \overline{\mathbf{y}}\right),
\end{array}
$$


where $\overline{\mathbf{y}}=S_{\ell} \mathbf{y}$ and $\bar{F}=S_{\ell} F$. Observe, that the Jacobian operator of $\bar{F}$ (with respect to $\overline{\mathbf{y}}$ ) can be obtained by deleting the first row and column of the matrix of the Jacobian operator of $F$. The tuple $\left(x_{0},\left(\mathbf{y}_{0}\right)_{1}, \overline{\mathbf{y}_{0}}\right)$ is a solution of (7) and (8). Set

$$
A=\frac{\partial F}{\partial \mathbf{y}}\left(x_{0}, \mathbf{y}_{0}\right) \quad \text { and } \quad B=\frac{\partial \bar{F}}{\partial \overline{\mathbf{y}}}\left(x_{0},\left(\mathbf{y}_{0}\right)_{1}, \overline{\mathbf{y}_{0}}\right) .
$$

Proposition 1 implies that $r(B)<r(A)=1$. Thus, we can again employ the Implicit Function Theorem and obtain that there exists a unique positive analytic solution $\overline{\mathbf{y}}=\overline{\mathbf{y}}\left(x, y_{1}\right)$ of (8) with $\overline{\mathbf{y}}(0,0)=\overline{\mathbf{0}}$ such that $\overline{\mathbf{y}}\left(x, y_{1}\right)$ is also defined in a neighborhood of $\left(x_{0},\left(\mathbf{y}_{0}\right)_{1}, \overline{\mathbf{y}_{0}}\right)$. Furthermore, we have $\overline{\mathbf{y}}\left(x_{0},\left(\mathbf{y}_{0}\right)_{1}\right)=$ $\overline{\mathbf{y}_{0}}$. If we insert this function into equation (7), we obtain a single equation

$$
y_{1}=F_{1}\left(x, y_{1}, \overline{\mathbf{y}}\left(x, y_{1}\right)\right)
$$

for $y_{1}=y_{1}(x)$. The function $G\left(x, y_{1}\right)=F_{1}\left(x, y_{1}, \overline{\mathbf{y}}\left(x, y_{1}\right)\right)$ is an analytic function around $(0,0)$ with $G\left(0, y_{1}\right)=0$ and such that all Taylor coefficients of $G$ are real and non-negative (this follows from the positivity of $F$ and $\left.\mathbf{y}\left(x, y_{1}\right)\right)$. Furthermore, the tuple $\left(x_{0},\left(\mathbf{y}_{0}\right)_{1}\right)$ belongs to the region of convergence of $G(x, y)$. In what follows, we show that $\left(x_{0},\left(\mathbf{y}_{0}\right)_{1}\right)$ is a positive solution of the system of equations

$$
\begin{aligned}
y_{1} & =G\left(x, y_{1}\right), \\
1 & =G_{y_{1}}\left(x, y_{1}\right),
\end{aligned}
$$

with $G_{x}\left(x_{0},\left(\mathbf{y}_{0}\right)_{1}\right) \neq 0$ and $G_{y_{1} y_{1}}\left(x_{0},\left(\mathbf{y}_{0}\right)_{1}\right) \neq 0$.

In order to see that $G_{y_{1}}\left(x_{0},\left(\mathbf{y}_{0}\right)_{1}\right)$ is indeed equal to 1 , note that the classical Implicit Function Theorem otherwise implies that there exists an analytic solution of $y_{1}=G\left(x, y_{1}\right)$ locally around $x_{0}$. Inserting this function into equation (8), we obtain that there also exists an analytic solution $\mathbf{y}(x)$ of (3) in a neighborhood of $x_{0}$. Implicit differentiation yields to

$$
\left(I-\frac{\partial F}{\partial \mathbf{y}}\left(x_{0}, \mathbf{y}_{0}\right)\right) \frac{\partial \mathbf{y}}{\partial x}\left(x_{0}\right)=\frac{\partial F}{\partial x}\left(x_{0}, \mathbf{y}_{0}\right) .
$$

Since the spectral radius of the (positive and irreducible) Jacobian operator at $\left(x_{0}, \mathbf{y}_{0}\right)$ is equal to 1 , there exists a strictly positive left eigenvector to the eigenvalue 1 ( $c f$. Section 4). Multiplying this vector to equation (9) from the left yields to a contradiction since $\frac{\partial F}{\partial x}\left(x_{0}, \mathbf{y}_{0}\right) \neq 0$ (note that $F(x, \mathbf{0}) \not \equiv \mathbf{0}$ and that $F$ is positive).

Next suppose that $G_{x}\left(x_{0},\left(\mathbf{y}_{0}\right)_{1}\right)=0$. The positivity implies that the unique solution of $y_{1}=G\left(x, y_{1}\right)$ is given by $y_{1}(x) \equiv 0$. Consider the solution $\mathbf{y}(x)$ of (3) for some real $x>0$ in the near of 0 . Since the spectral radius of the Jacobian operator is smaller than 1 (for $x$ small), we can express the resolvent with the aid of the Neumann series, i.e., we have (cf. (9))

$$
\frac{\partial \mathbf{y}}{\partial x}(x)=\left(I-\frac{\partial F}{\partial \mathbf{y}}(x, \mathbf{y}(x))\right)^{-1} \frac{\partial F}{\partial x}(x, \mathbf{y}(x))=\sum_{n \geqslant 0}\left(\frac{\partial F}{\partial \mathbf{y}}(x, \mathbf{y}(x))\right)^{n} \frac{\partial F}{\partial x}(x, \mathbf{y}(x)) .
$$

Since $\partial F / \partial \mathbf{y}$ is irreducible and $\partial F / \partial x \neq 0$ we obtain that $y_{1}(x)$ cannot be a constant function. Finally, the positive function $G$ cannot be an affine map in $y$ since otherwise the conditions $G\left(0, y_{1}\right)=0$ and $G\left(x_{0},\left(\mathbf{y}_{0}\right)_{1}\right)=\left(\mathbf{y}_{0}\right)_{1}$ imply that $y_{1}(x)$ is constant. 
If follows from [Drm09, Theorem 2.19] that there exists a unique solution $\tilde{y}\left(x_{1}\right)$ of the equation $y_{1}=$ $G\left(x, y_{1}\right)$ with $\tilde{y}_{1}(0)=0$. It is analytic for $|x|<x_{0}$ and there exist functions $g_{1}(x)$ and $h_{1}(x)$ that are analytic around $x_{0}$ such that $\tilde{y}_{1}(x)$ has a representation of the form

$$
\tilde{y}_{1}(x)=g(x)-h(x) \sqrt{1-\frac{x}{x_{0}}}
$$

locally around $x_{0}$. Due to the uniqueness of the solution $y(x)$ of the functional equation (3), we have that the first component of $y(x)$ coincide with $\tilde{y}_{1}(x)$, i.e., $y_{1}(x)=\tilde{y}_{1}(x)$. Inserting this solution into equation (8) (note, that $\bar{F}$ is analytic at $\left.\left(x_{0},\left(\mathbf{y}_{0}\right)_{1}, \overline{\mathbf{y}_{0}}\right)\right)$, we obtain $\overline{\mathbf{y}}\left(x, y_{1}(x)\right)=\left(y_{2}(x), y_{3}(x), \ldots\right)$. Thus, $y_{j}(x)$ has a representation of the form (10) for all $j \geqslant 1$. This finally shows (4).

If there exist two integers $n_{1}$ and $n_{2}$ that are relatively prime such that $\left[x^{n_{1}}\right] y_{1}(x)>0$ and $\left[x^{n_{2}}\right] y_{1}(x)>$ 0 , then it follows that $x_{0}$ is the only singularity of $y_{1}(x)$ on the circle $|x|=x_{0}$ and there exists an analytic continuation to a $\Delta$-domain. Indeed, it suffices to show that

$$
G_{y_{1}}\left(x, y_{1}\right)<1
$$

for $|x|=x_{0}$ but $x \neq x_{0}$ (compare with the proof of [Drm09, Theorem 2.19]). Since $y_{1}(x)$ is positive, we clearly have $\left|y_{1}(x)\right| \leqslant y_{1}(|x|)$. If equality occurs, then

$$
x^{n_{1}}=|x|^{n_{1}}=x_{0}^{n_{1}} \quad \text { and } \quad x^{n_{2}}=|x|^{n_{2}}=x_{0}^{n_{2}} .
$$

By assumption, $n_{1}$ and $n_{2}$ are relatively prime and we obtain $x=x_{0}$, which is impossible. Thus, we actually have $\left|y_{1}(x)\right|<y_{1}(|x|)$. The positivity of $G$ implies

$$
\left|G_{y_{1}}\left(x, y_{1}(x)\right)\right| \leqslant G_{y_{1}}\left(|x|,\left|y_{1}(x)\right|\right)<G_{y_{1}}\left(|x|, y_{1}(|x|)\right)=G_{y_{1}}\left(x_{0},\left(\mathbf{y}_{0}\right)_{1}\right)=1 .
$$

Inserting the analytic continuation of $y_{1}(x)$ into equation (8), we obtain that there exists an analytic continuation for all $\mathbf{y}_{j}$. The Transfer Lemma of Flajolet and Odlyzko implies that $\left[x^{n}\right] y_{j}(x)$ satisfy

$$
\left[x^{n}\right] y_{j}(x) \sim \frac{c_{j}}{x_{0}^{n} n^{3 / 2}}
$$

for all $j \geqslant 1$ and for some constants $c_{j}>0$. This finally shows Theorem 2 .

Acknowledgments. The author would like to thank Michael Drmota for his valuable advices and Bernhard Gittenberger and Michael Kaltenbäck for helpful discussions.

\section{References}

[BBY06] Jason P. Bell, Stanley N. Burris, and Karen A. Yeats. Counting rooted trees: the universal law $t(n) \sim C \rho^{-n} n^{-3 / 2}$. Electron. J. Combin., 13(1):Research Paper 63, 64 pp. (electronic), 2006.

[BBY09] Jason P. Bell, Stanley N. Burris, and Karen A. Yeats. Characteristic points of recursive systems. arXiv:0905.2585v1 [math.CO], 2009. 
[BDFG03] Jérémie Bouttier, Philippe Di Francesco, and Emmanuel Guitter. Geodesic distance in planar graphs. Nuclear Phys. B, 663(3):535-567, 2003.

[BM06] Mireille Bousquet-Mélou. Limit laws for embedded trees: applications to the integrated superBrownian excursion. Random Structures Algorithms, 29(4):475-523, 2006.

[Dei85] Klaus Deimling. Nonlinear functional analysis. Springer-Verlag, Berlin, 1985.

[Drm97] Michael Drmota. Systems of functional equations. Random Structures Algorithms, 10(12):103-124, 1997. Average-case analysis of algorithms (Dagstuhl, 1995).

[Drm09] Michael Drmota. Random trees. SpringerWienNewYork, Vienna, 2009. An interplay between combinatorics and probability.

[FO90] Philippe Flajolet and Andrew Odlyzko. Singularity analysis of generating functions. SIAM J. Discrete Math., 3(2):216-240, 1990.

[FS09] Philippe Flajolet and Robert Sedgewick. Analytic combinatorics. Cambridge University Press, Cambridge, 2009.

[Kat66] Tosio Kato. Perturbation theory for linear operators. Die Grundlehren der mathematischen Wissenschaften, Band 132. Springer-Verlag New York, Inc., New York, 1966.

[Knu75] Donald E. Knuth. The art of computer programming. Addison-Wesley Publishing Co., Reading, Mass.-London-Amsterdam, second edition, 1975. Volume 1: Fundamental algorithms, Addison-Wesley Series in Computer Science and Information Processing.

[KR50] M. G. Kreı̌n and M. A. Rutman. Linear operators leaving invariant a cone in a Banach space. Amer. Math. Soc. Translation, 1950(26):128, 1950.

[La193] Steven P. Lalley. Finite range random walk on free groups and homogeneous trees. Ann. Probab., 21(4):2087-2130, 1993.

[Lal01] Steven P. Lalley. Random walks on regular languages and algebraic systems of generating functions. In Algebraic methods in statistics and probability (Notre Dame, IN, 2000), volume 287 of Contemp. Math., pages 201-230. Amer. Math. Soc., Providence, RI, 2001.

[Lal02] Steven P. Lalley. Random walks on infinite free products and infinite algebraic systems of generating functions. http://www.stat.uchicago.edu/lalley/Papers/index.html, 2002.

[Pro04] Helmut Prodinger. The kernel method: a collection of examples. Sém. Lothar. Combin., 50:Art. B50f, 19 pp. (electronic), 2003/04.

[VJ67] David Vere-Jones. Ergodic properties of nonnegative matrices. I. Pacific J. Math., 22:361$386,1967$.

[Woo97] Alan R. Woods. Coloring rules for finite trees, and probabilities of monadic second order sentences. Random Structures Algorithms, 10(4):453-485, 1997.

[Zei86] Eberhard Zeidler. Nonlinear functional analysis and its applications. I. Springer-Verlag, New York, 1986. Fixed-point theorems, Translated from the German by Peter R. Wadsack. 
